\title{
A Reaction Network for Prebiotic Synthesis
}

\section{ALBERT C FAHRENBACH}

University of New South Wales

Presenting Author: a.fahrenbach@unsw.edu.au

Complex abiotic reaction networks on the early Earth, capable of producing biologically important molecules like nucleic acids and peptides are suspected to have underpinned life's emergence. Experimental models for these reaction networks are currently undergoing rapid development. In this online seminar, a reaction network[1] that produces precursors for ribonucleotide and amino acid synthesis will be described. The reaction network starts from an aqueous mixture of $\mathrm{NaCl}, \mathrm{NH}_{4} \mathrm{Cl}, \mathrm{P}_{\mathrm{i}}$ and $\mathrm{HCN}$ as the only source of carbon. This initial mixture is then made to evolve chemically by a combination of $\gamma$-radiolysis and drydown. No well-timed addition of any other reagents is required. This continuous reaction network can help us understand how important biomolecules like RNA and peptides might arise spontaneously from a complex mixture in a manner that minimizes human intervention.

[1] Yi, Tran, Ali, Yoda, Adam, Cleaves \& Fahrenbach (2020), Proc. Natl. Acad. Sci. USA 117, 13267-13274.

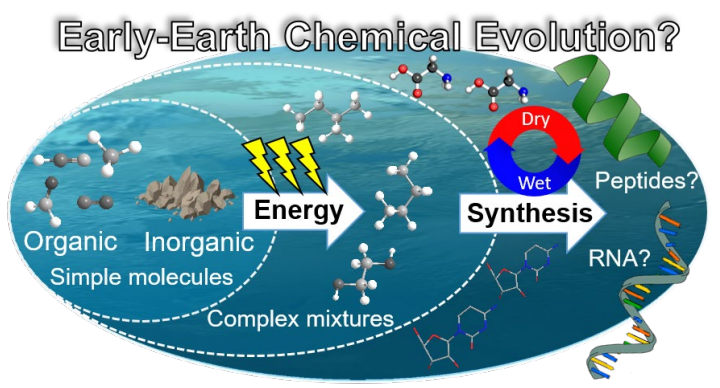

